\title{
Adipose Stem Cell Effect on Renal Oxidative Stress in Chronic Nephropathy Rat Model
}

\author{
Asmaa A. Seddek, Magda I. Mohamed, Angie M. Ameen, and \\ Noha M. Abogresha*
}

Department of Physiology, Faculty of Medicine, Suez Canal University, Egypt

\begin{abstract}
Background: Chronic kidney disease (CKD) is a worldwide health problem, and the number of affected individuals is steadily increasing over time. It has been suggested that mesenchymal stem cells can be used as a novel treatment option for the management of CKD, based on encouraging data from rodent studies. Adipose-Derived (ADMSCs) are attractive sources of stem cells which have the capacity to differentiate into other cell types such as adipocytes, myocytes, osteoblasts, and neurons. Aim: The present study aimed to elucidate the potential role of adipose stem cells in the treatment of chronic kidney diseases. Oxidative stress was evaluated besides the histopathological findings. Subjects and Methods: Three groups of rats (10 rats each) were studied for 12 weeks after induction of CKD in 2 groups one of them treated with ADMSCs, Oxidative stress was measured in kidney tissue. Results: The oxidative stress capacity of the kidney was better in rats treated with ADMSCs than in the non-treated group. Histopathological examination revealed amelioration of renal tissue on treatment with adipose stem cells. Expression of mitochondrial DNA was also improved. Conclusion: ADMSCs can reduce the severity of the renal injury and prevent the progression of renal fibrosis after injury through suppressing oxidative stress
\end{abstract}

Keywords: Chronic kidney disease, Adipose mesenchymal stem cells

\section{Introduction}

In Egypt, there are no recent data about the prevalence of ESRD; however, the last statistics was performed in 2004, with a prevalence of $483 \mathrm{ppm}$ (patient per million $)^{(1)}$. It is defined as reduction in kidney function, demonstrated by decreased glomerular filtration rate (GFR), or evidence of kidney damage, such as increased excretion of urinary albumin, increased serum creatine or urea(2). MSCs are pluripotent, long, shuttle-shaped, fibroblast like cells that are presented in almost all body tissues, including the bone marrow, periosteum, fat tissue, pulp, synovium, umbilical cord, placenta, amniotic fluid and fetal tissues ${ }^{(3)}$ Thus, MSCs become the preferred seeds for tissue engineering. After systemic infusion, MSCs can preferentially migrate into sites of inflammation and injury $(4,5)$. ADMSCs have the advantages of minimal invasiveness of harvesting and plentiful supply from culturing. These cells

*Corresponding Author: Noha_ibrahim@med.suez.edu.eg 
have no ethical problem regarding the source and less concern about allo- and xenografting. Most importantly, ADMSCs have more potent anti-inflammatory and immune-modulating functions than $\mathrm{BMSCs}^{(6)}$. Structural characteristics in CKD, loss of renal energy, and uremia result in an imbalance between free radical production and antioxidant defenses. Oxidative stress because of the increase in reactive oxygen species (ROS) and decrease in antioxidant defenses is prevalent in many health problems like CKD and it still exists after transplantation. Oxidative compounds have physiologic defense mechanisms in the body, but imbalance in oxidant generation results in tissue damage. Oxidative stress induces endothelial dysfunction and progression of atherosclerosis in patients with $\mathrm{CKD}^{(7)}$. Combining the above facts together, the present study hypothesized that administration of adipose derived mesenchymal stem cells could potentially reverse this scenario. Therefore, the current study evaluated the antioxidant effect of adipose derived mesenchymal stem cells in adriamycin induced chronic renal failure in rats.

\section{Materials and Methods}

\section{Animals}

Thirty male white albino rats of local strain with an average weight of (200 $20 \mathrm{gm}$.) were selected and included in this study, they were brought from the national research center, Cairo. Animals were housed in spacious plastic cages at controlled room temperature and were kept with free access to standard rat chow diet and tap water. They were left for acclimatization for one week before the start of the study. Animal's care before and during the experimental procedures was done in accordance with the guidelines of the Animal Ethical Committee, Faculty of Medicine, Suez Canal University. The study was approved by the Animal Research Ethics Committee of the colleague. After acclimatization rats were randomly divided into 3 groups, 10 rats per group where Group 1: (Normal group), the rats received $0.5 \mathrm{~mL}$ saline as a single intravenous injection (in the tail vein). Group 2: (Adriamycin group), the rats received a single intravenous injection (tail vein) of ADR at a dose of $5 \mathrm{mg} / \mathrm{kg}$ of body weight $^{(8)}$. Group 3: (AD-MSCs group), rats received AD-MSCs $(2 \times 106$ cells suspended in $1 \mathrm{ml}$ saline) one week after the ADR injection $^{(9)}$.

\section{Chemicals}

1-Drugs:

Doxorubicin hydrochloride (ADR) was purchased in the form of vials ( $50 \mathrm{mg} / 25 \mathrm{~mL}$ saline) from Oncology Unit Suez Canal University Hospital, Saline.

\section{2-Reagents:}

A) Reagents for stem cell adipose separation and culture: Collagenase type I (cat. no. C-0130, Sigma-Aldrich), Hanks Balanced Salt Solution (HBSS, without phenol red; cat. No. 14025-050, Gibco- BRL), Fetal bovine serum FBS (FBS, cat. no. F6178, sigma-Aldrich), Phosphate buffered saline (PBS) (Lonza-belgium, cat. No. BE17-516F), Dulbecco's modified Eagle's medium (DMEM) Low Glucose, w/L-Glutamine, $w / S o d i u m$ Pyruvate (Biowest, cat. No. L0060500), Streptomycin, penicillin (p/s) (cat. No. P4333, Sigma-Aldrich), Trypsin (Lonza,Walkersville, cat. No. D2287), Feridex (ferumoxides).

B) Reagents for lipid peroxidation malondialdyhyde (MDA) and total antioxidants in kidney tissues: Rat Malondialdehyche, MDA ELISA Kit (Cat. No. BYEK1371, Biopes), Total Antioxidant Capacity, TAC Assay Kit (Cat. No. BYEK2200, Biopes).

C) Reagents for histopathology: Hematoxylin and Eosin (H\&E) and Masson's Trichrome dye used for histopathological evaluation of renal tissue, Prussian blue stain for detection of iron labeled stem cells.

\section{Methods}

I-Induction of chronic nephropathy: Nephropathy was induced by a single intravenous (I.V) injection of ADR at a dose of 5 $\mathrm{mg} / \mathrm{kg}$ of body weight ${ }^{(8)}$. ADR was purchased in vials ( $50 \mathrm{mg} / 25-\mathrm{mL}$ saline) from EIMC United Pharmaceuticals (EUP), Badr City, Cairo, Egypt. 


\section{II- Collection and preparation of ADSC}

Cell source: Adipose tissue was isolated from rat's peritoneum, (rat peritoneal adipose tissue).

\section{1- Separation of adipose tissue stem cells}

A- Isolation of adipose tissue

Adipose tissue was isolated from apparently healthy rats after scarification. It was collected in a sterile bottle that contains equal volume of phosphate buffer saline (PBS) with 1-5\% penicillin /streptomycin/ Amphotericin. Supernatant is removed unntil became clear; then transferred to a Petri dish and minced using scissors and scalpel into small parts to ease digestion.

\section{$B$ - Digestion with Collagenase}

About $2 \mathrm{ml}$ Collagenase IV prepared at 1-2 $\mathrm{mg} / \mathrm{mL}$ was added to a sterile Petri dish containing minced adipose tissue. The mixture was left then neutralized by adding DMEM medium containing $10 \%$ heat inactivated fetal bovine serum. The sample was pipetted several times to aid disintegration then transferred to $15 \mathrm{ml}$ sterile falcon tubes (large pieces should be avoided). Centrifugion at $2000 \mathrm{rpm}$ for $5 \mathrm{~min}$ was done to get the stromal vascular fraction (containing adipose stem cells). The suspension was transferred to culture flask and incubated at $37^{\circ} \mathrm{C}$ and $5 \% \mathrm{CO} 2$ for 72 hours ${ }^{(10)}$.

2- Culture of mesenchymal stem cell(11)

Cells were cultured at a density of $\left(1 \times 10^{6}\right)$ mononuclear cells $/ \mathrm{cm}^{2}$. Cells were nurtured with culture medium DMEM supplemented with $20 \%$ fetal bovine serum, $1 \%$ antibiotic/antimycotic, and $1 \%$ glutamine. Cultures were incubated at $37^{\circ} \mathrm{C}$, humidified atmosphere containing $5 \% \mathrm{CO}_{2}$. After overnight incubation, non-adherent cells were removed, and fresh medium was added to the culture flask. Later, the culture medium was changed at every four days and cellular growth assessed daily under an inverted microscope. When the cells reached $50-60 \%$ confluence, they were harvested after trypsin/ EDTA (ethylene-diaminetetra-acetic acid) (0.025\%).

\section{3- Labeling of MSCS}

Before cell transplantation, the cultured cells were impregnated with an injectable solution of ferumoxides 25microgramFe/ ml (Feridex) (Bayer Health Care Pharmaceuticals) for 24 hours with 375 nanogram $/ \mathrm{ml}$ poly L lysine added 1 hour before cell incubation. Feridex labeled MSCs were washed in PBS, trypsinized, washed and resuspended in $0.01 \mathrm{Mol} / \mathrm{L}$ PBS at concentration of $1 \times 1,000,000$ cells $/ \mathrm{ml}$. Labeling was histologically assessed using Prussian blue ${ }^{(11)}$.

\section{III- Assessment of renal function tests}

Retro-orbital blood samples were taken then placed into tubes (EDTA-containing tubes). Blood samples were collected from the tail vein and kept on ice for 1 hour. Serum was separated by centrifugation at $2000 \mathrm{rpm}$ for $15 \mathrm{~min}$. and stored at $-80^{\circ} \mathrm{C}$ until chemical analysis of serum urea, serum albumin and creatinine by an automatic chemistry analyzer (Hitachi, Japan). At the end of the study, (week 12) rats were sacrificed by cervical decapitation; blood samples were taken by heart puncture and placed into EDTA-containing tubes).

\section{IV- Urine samples}

Metabolic cages used for the collection of urine samples. Proteinuria was determined by using an automatic biochemistry analyzer (DADE Xpand, USA). Proteinuria was expressed as milligrams (mg) per $24 \mathrm{~h}$. It was assessed for all groups at Day 0, day 7 as Adriamycin start kidney injury two days after Adriamycin injection and kidney injury become prominent four days after Adriamycin injection, weeks 4, 8 and by the end of the study at week 12 .

V-Assessment of lipid MDA and total antioxidant capacity in kidney tissues

The kidney was weighed and homogenized in $5^{-10} \mathrm{~mL}$ cold buffer $(50 \mathrm{mmol} / \mathrm{L}$ potassium phosphate, $\mathrm{pH} 7.5,1 \mathrm{mmol} / \mathrm{L}$ EDTA) per gram of tissue, using a mortar and pestle, then centrifuged at $1000 \mathrm{x} g$ for $15 \mathrm{~min}$ 
at $4^{\circ} \mathrm{C}$. The supernatant was kept at $-80^{\circ} \mathrm{C}$ for analysis of lipid peroxides (MDA) and TAO. TAO was measured using a colorimetric assay kit (sigma, Egypt) according to the manufacturer's instructions. MDA was measured by ELISA technique.

VI- Quantification of mitochondrial DNA copy number in renal tissue

For quantification of the mtDNA copy number, total DNA was extracted and compared with the relative amounts of mtDNA and nuclear DNA contents. Relative amounts of mtDNA and nuclear DNA were determined by $\mathrm{qPCR}$ (quantitative polymerase chain reaction)

\section{VII- Histopathology of renal tissue}

At the end of the study, rats were anaesthetized and the kidneys were removed. The right kidney tissue was perfused with Hanks' buffer to eliminate the intravascular blood content and then fixed in 10\% formaldehyde for 24 hours, dehydrated by gradient ethanol, embedded in paraffin, and sectioned at $4 \mu \mathrm{m}$ thicknesses. Renal sections were stained with H\&E and Masson Trichrome staining. Assessment of renal damage was done without prior knowledge of the group to be assessed (blind assessment). All Images were captured for histopathology using: Calibrated standard digital microscope camera (Tucsen ISH1000 digital microscope camera) using Olympus ${ }^{\circledR} \mathrm{CX}_{21}$ microscope, with resolution of 10 MP (megapixels) (3656 x 2740 pixel each image). All slides were captured with Power Field of 400 x, UIS optical system (Universal Infinity System, Olympus ${ }^{\circledR}$, Japan).

a) Evaluation of renal tubular damage Evaluation of renal tubule-interstitial damage was scored by a semi-quantitative method; histological samples were scored by a blinded examiner. In brief, the degree of renal tubular damage was scored as Follows: 0, normal; 1 , very mild focal dilatation, the area of interstitial inflammation and fibrosis, tubular atrophy and dilation involving $<25 \%$ of the field; 2, large number of dilated tubules with widening of the interstitium, sclerotic lesion area between $25-50 \%$ of the field; and 3, fairly extensive dilatation of tubules and widening of the interstitium with lesions involving $>50 \%$ of the field and 4, complete atrophy of the tubules. The individual scores were then averaged for each group according to the previously described scoring system ${ }^{(12)}$.

b) Evaluation of the degree of fibrosis:

Fibrosis was determined by the areastained blue in each field and was given a grade. In all tissue samples, 10 to 15 fields were analyzed for glomerular fibrosis determination. Glomerular fibrosis was scored as follows:(12): 1= fibrosis $<25 \%$ / 100 fields; $2=$ fibrosis $25 \%-<50 \%$ $/ 100$ fields; $3=$ fibrosis $50 \%-<75 \% / 100$ fields; $4=$ fibrosis $\geq 75 \% / 100$ fields

\section{Statistical Analysis}

Data were expressed as mean \pm SD and were analyzed using one-way ANOVA and post-hoc Tukey test. Data were analyzed using statistical package for social sciences (SPSS) software version 16 (SPSS Inc., Chicago, IL, USA).

\section{Results}

Thirty adult male albino rats were evaluated and divided into three groups (10 rats each); G1: normal group, G2: chronic nephropathy, and G3: adipose stem cells. The follow-up period was 12 weeks, ended by scarification of all rats and the tissue was sent for pathologic, PCR, and oxidative stress markers evaluation.

\section{Assessment of animal body weight}

ADR causes a decrease in body weight in all study groups when compared to normal ( $p$ $\leq 0.05$ ) in groups of chronic nephropathy \& 
ADSCs. Treatment with ADSCs caused significant increase in body weight when compared to chronic nephropathy group ( $p \leq 0.05$ ) (figure 1).

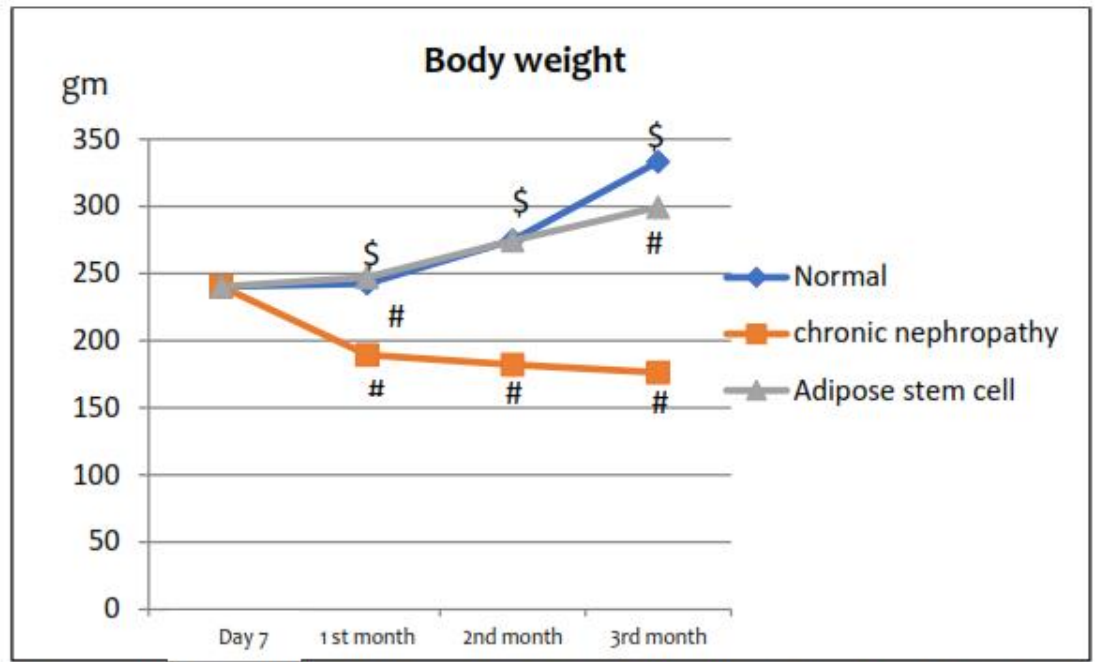

Figure 1: Body weight in all study groups at base line, $1^{\text {st }}, 2^{\text {nd }}$, and $3^{\text {rd }}$ month

(\#) significant $P$ value compared to normal group and (\$) significant $P$ value compared to $C h$. nephropathy)

2. Assessment of urinary protein output ADR causes an increase in urinary protein in chronic nephropathy \& ADSCs groups compared to normal $(p \leq 0.05)$ Treatment with ADSCs caused significant decrease in urinary protein compared to chronic nephropathy group $(p \leq 0.05)$ Figure ( 2$)$.

\section{Assessment of renal function}

Serum albumin: ADR caused a decrease in serum albumin in all study groups when compared to normal ( $p \leq 0.05)$. Treatment with ADSCs caused significant increase in serum albumin when compared to chronic nephropathy group ( $p \leq 0.05$ ) Figure (3).
Serum creatinine: ADR effect on serum creatinine was a significant increase in second \& third groups when compared to normal ( $p$ s0.05). Treatment with ADSCs caused significant decrease in serum creatinine when compared to chronic nephropathy group ( $p \leq 0.05$ ). (Figure 4).

Serum urea: Serum urea levels were significantly elevated by ADR in groups (chronic nephropathy, ADSCs) compared to normal ( $p \leq 0.05)$. ADSCs treatment caused a significant decrease in serum urea when compared to chronic nephropathy group ( $p$ $\leq 0.05$ ). (Figure 5)

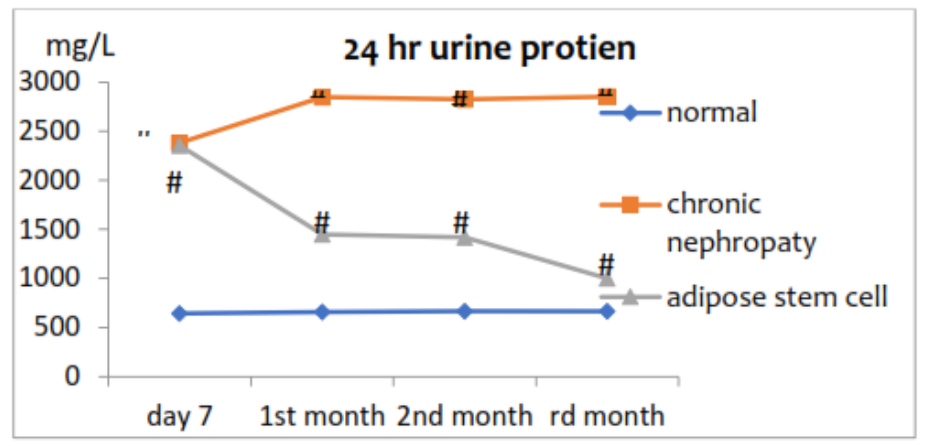

Figure 2: $24 \mathrm{hr}$. Urine protein in all study groups

(\#) significant $P$ value compared to normal group and (\$) significant $P$ value compared to Ch. nephropathy) 


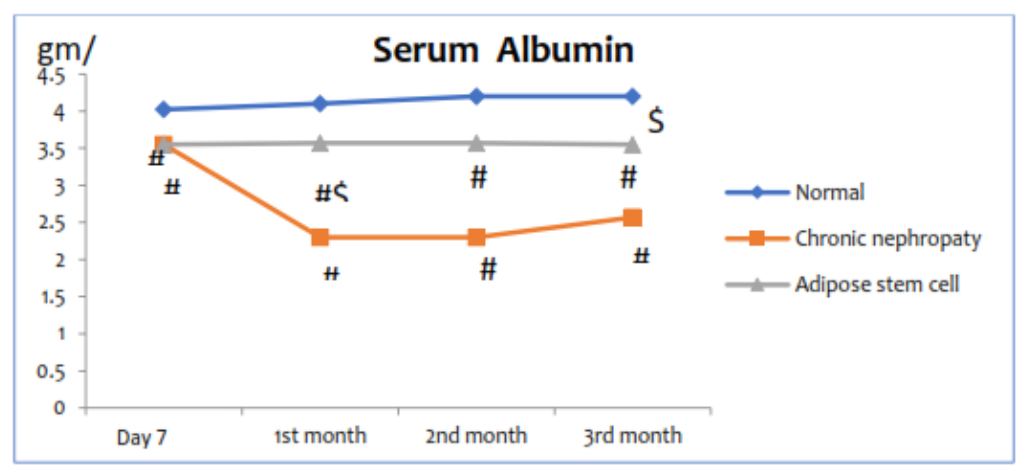

Figure 3: Serum albumin levels in all study groups

(\#) significant $P$ value compared to normal group and (\$) significant $P$ value compared to Ch. nephropathy)

4. Assessment of oxidative stress

Total antioxidant: Figure 6 showed decreased TAO capacity in study groups under the effect of ADR in Adipose stem cells and chronic nephropathy groups. Results were significant when compared to normal group ( $p \leq 0.05)$. ADSC s treated groups was significantly higher than chronic nephropathy group ( $\mathrm{p} \leq 0.05)$.

Lipid peroxidase malondi-aldyhyde (MDA): Figure 7 showed increased renal MDA in study groups under the effect of ADR \& Adipose stem cell groups. Results were significantly higher when compared to normal group ( $p \leq 0.05)$. Treated group was significantly lower than chronic nephropathy group ( $\mathrm{p} \leq 0.05)$.
5. Mitochondrial DNA copy number

Adipose treated and chronic nephropathy groups results were significantly lower than normal group ( $p \leq 0.05)$. Treated group with ADSCs was significantly higher compared to chronic nephropathy group ( $p \leq 0.05)$. (figure 8).

\section{Histopathological scoring}

ADR significantly increased tubular necrosis, inflammatory cell infiltration and interstitial fibrosis and significantly decreased regenerating tubules in chronic nephropathy \& ADSCs compared to normal group ( $p$ $\leq 0.05$ ). Treated group with ADSCs had significant decrease in these changes compared to chronic nephropathy. (figure 9).

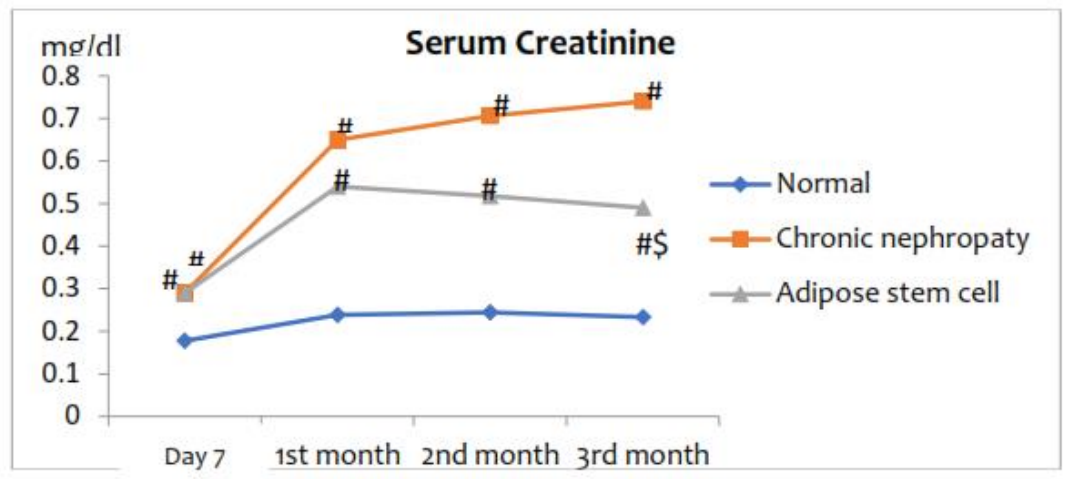

Figure 4: Serum creatinine levels in all study groups

(\#) significant $P$ value compared to normal group and (\$) significant $P$ value compared to Ch. nephropathy) 


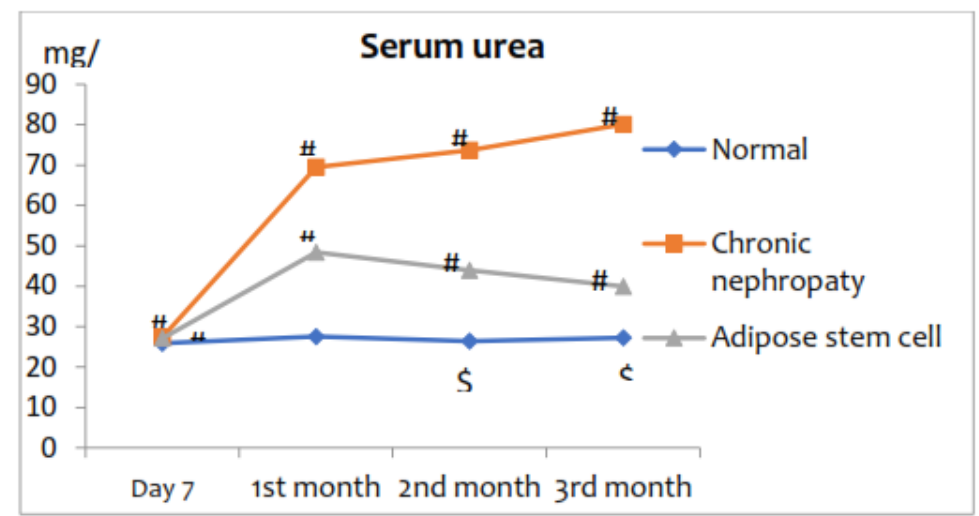

Figure 5: Serum urea levels in all study groups

(\#) significant $P$ value compared to normal group and (\$) significant $P$ value compared to Ch. nephropathy)

Hematoxylin and Eosin staining

H\&E staining of normal group sections showed kidney tissue formed of glomeruli and tubules, glomeruli showed capillary tuft, thin Bowman's space and mesangial cells, variable sized tubules lined by columnar cells with eosinophilic cytoplasm and central vesicular nucleus \& interstitium showed thin-walled blood vessels and scant stroma. No inflammation signs (Figure 10a). H\&E staining of chronic nephropathy group showed marked interstitial inflammatory infiltrate formed mainly of lymphocytes with markedly congested thick-walled vessels, tubular epithelial cells showed desquamation and hydropic degeneration with tubular necrosis, with few regenerating tubules (Figure 10b). While H\&E staining of adipose treated group showed marked improvement of changes presented as decreased inflammation and many regenerating tubules with minimal hydropic degeneration glomerular tuft (Figure 10c).

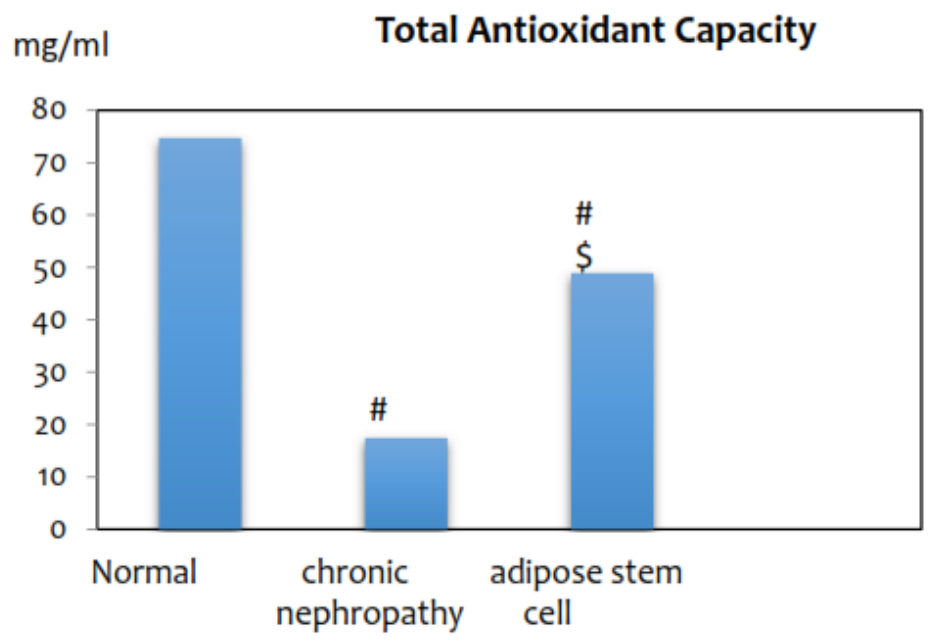

Figure 6: Renal total antioxidant capacity in all study groups

(\#) significant $P$ value compared to normal group and (\$) significant $P$ value compared to Ch. nephropathy 


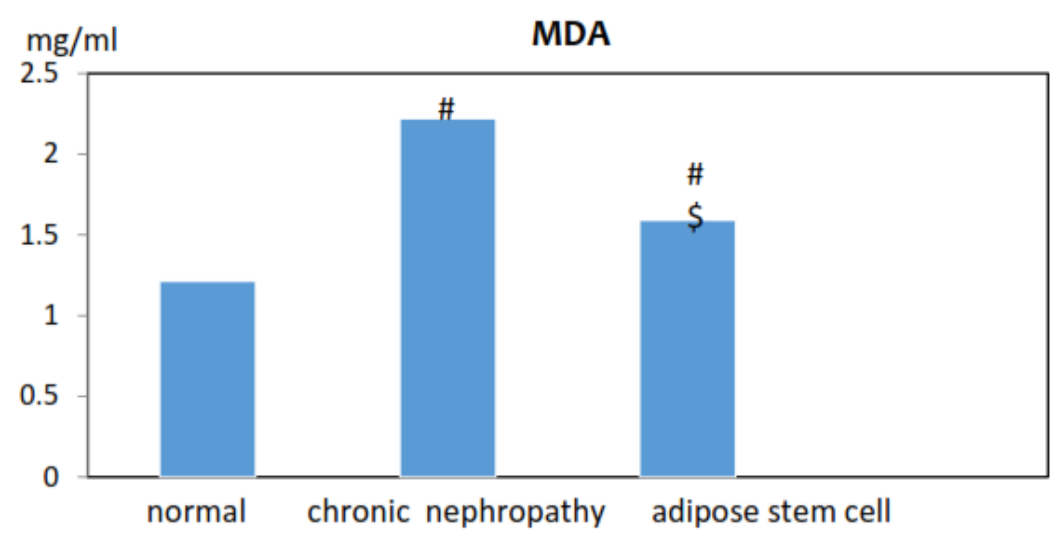

Figure 7: Renal malondialdyhyde in all study groups

(\#) significant $P$ value compared to normal group and (\$) significant $P$ value compared to Ch. nephropathy)

\section{Masson's trichrome staining}

Masson's trichrome staining of renal tissue is shown in figure 11. Image analysis of Masson trichome staining included Evaluation of the degree of fibrosis: Fibrosis was determined by the area-stained blue in a given field, in chronic nephropathy group ADR effect on renal structure is shown in (figure 12) as significantly the highest area stained when compared to normal \& ADSC groups $(p \leq 0.05)$ in the mentioned groups.
Prussian blue stain for stem cell homing Using Prussian blue stain for stem cell homing, chronic nephropathy group showed negative staining (Figure 13). While, ADSC group showed positive staining.

Image analysis

ADSCs homing was higher in renal tissue of adipose stem cell group compared to normal \& chronic nephropathy groups ( $\mathrm{p}$ $\leq 0.05$ ) (figure 14).

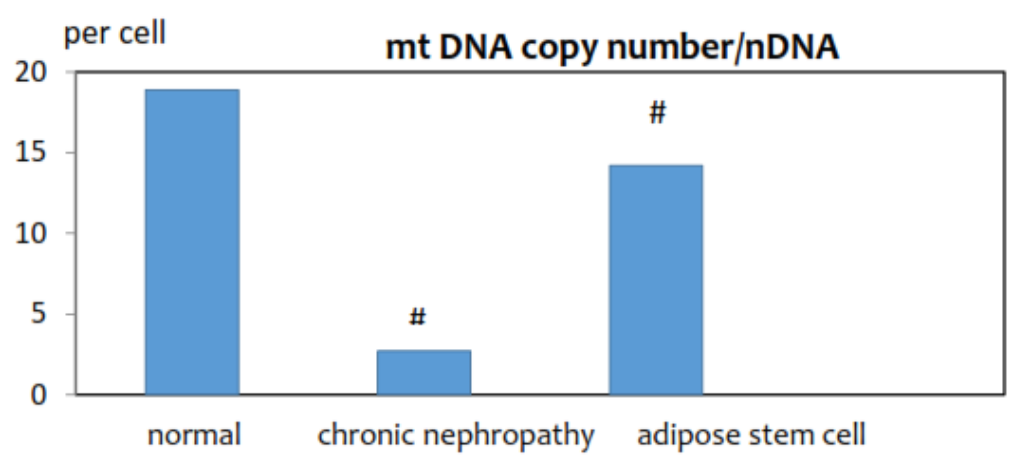

Figure 8: Mitochondrial DNA copy number in renal tissue in all study groups

(\#) significant $P$ value compared to normal group and (\$) significant $P$ value compared to Ch. nephropathy)

\section{Discussion}

The current study addressed the possible therapeutic effect of ADSC therapy on oxidative stress. ADR induced glomerular damage. ADR is a commonly used chemotherapeutic drug that was used to induce a model of nephropathy in albino rat. This 
model was evidenced by histological, histochemical, immunohistochemical, morphometric and serological studies(13). In this study we tested the effect of injected adipose on renal structure and function in ADR nephropathy in rats. Concerning the functional status of kidneys in our study heavy proteinuria and low serum albumin was observed in non- treated group. This agreed with other studies that evidenced heavy proteinuria after short term observations in ADR induced nephropathy in rats. ${ }^{14}$ ) Also, other study established the improvements in proteinuria in CKD rats injected with stem cells(4). Serum urea and creatinine were increased significantly in ADR CKD group than stem cell treated groups. This was in agreement with a study that reported MSCs induced functional improvement proved by reduction in plasma creatinine level(15). The functional improvement we proved was opposed by a study which found that in all cases there was no effect of MSCs on any of the clinical parameters that are classically utilized to monitor ADR-induced nephrosis, including 24-h proteinuria, renal function ${ }^{(16)}$. Also, it was posted that MSC administration failed to cause significant improvements in animal survival rate, body mass, serum creatinine, serum albumin, urinary protein excretion $^{(12)}$. In that study CKD was induced by 2 i.v. injections of ADR ( $4 \mathrm{mg} A D R / \mathrm{kg}$ body mass) into the penile vein at 14-day intervals and in MSC treated group; rats received 2 i.v. injections of MSCs $(2 \times 106$ cells) at 14-day intervals ( 5 days after each ADR injection). Concerning renal oxidative capacity (renal total antioxidant capacity and MDA) results in our study adriamycin CKD non treated group had elevated renal oxidative stress that is supported by studies ${ }^{(14)}$ in which Adriamycin was found to reduce glomerular ATPase activity, depleted levels of glutathione (antioxidant) and elevate levels of lipid peroxide in kidney. And, another study clarified that the major cause of the doxorubicin CKD model has been attributed to the oxidative injury evoked by a formation of doxorubicin-iron complexes(15). Moreover, the treated groups had better improvement than untreated which is similar to results in other study (12) that MSC caused significant improvement in renal oxidative stress markers which was demonstrated by significant reductions in oxidative stress in kidney tissues (as evidenced by reduction in MDA and elevation of GSH in MSCs groups) and also in agree with other studies that posted that MSCs are protective against cell damage by free radicals, provided that a sufficient number come in contact with the source of radicals and/or the damaged tissue ${ }^{(17)}$. Other studies opposed our findings ${ }^{(6,18)}$ postulated that the uremic toxins produced in patients with nephropathy, may inhibit differentiation and function of BM-MSCs. Also another study stated that complications of CKD, such as secondary hyperparathyroidism or lack of erythropoietin, may also contribute to dysfunction of $B M-M S C s^{(18)}$. Here nephropathy done by NPX (5/6 nephrectomy), SCs derived from adult human adipose tissue before stem cell injection adipogenic differentiation was done to isolated cells by supplementing the medium with $1 \times 10-6$ dexamethasone, $0.02 \mathrm{mg} / \mathrm{ml}$ indomethacin and 10 $\mu \mathrm{g} / \mathrm{ml}$ insulin, rats injected in the tail vein with ( $0.5 \times 106$ cells) of AD-MSCs, Rats were killed after 35 days.

\section{Conclusion}

ADSCs-treated group showed improved renal structure and function evidenced by better histopathology and oxidative capacity. MSC therapy for kidney injury is very promising with good safety profiles. MSC has the advantage of relatively easy isolation and culture conditions, making them a potentially low-cost and personalized therapy option for patients with kidney injury. 
A

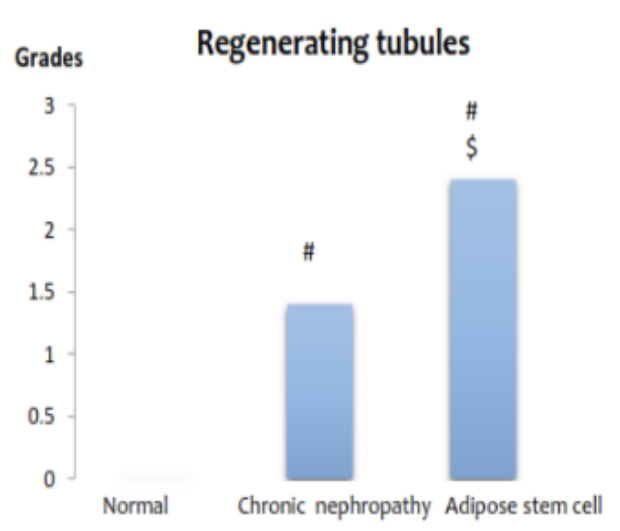

C

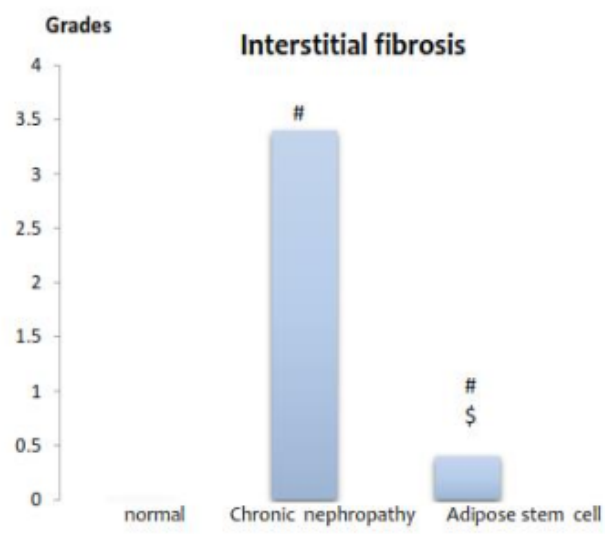

B

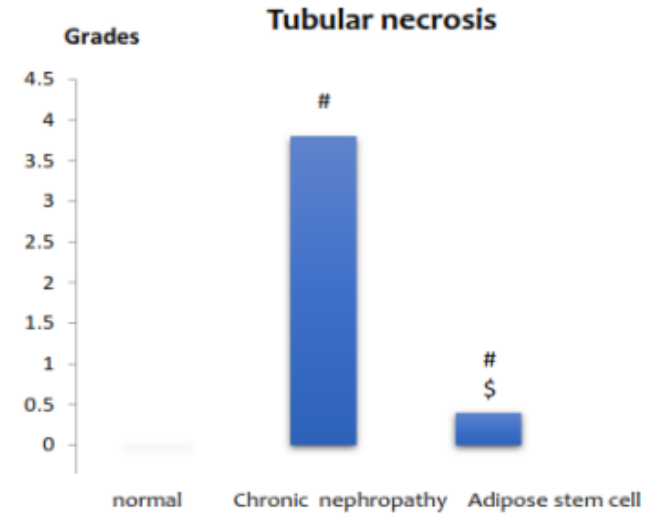

D

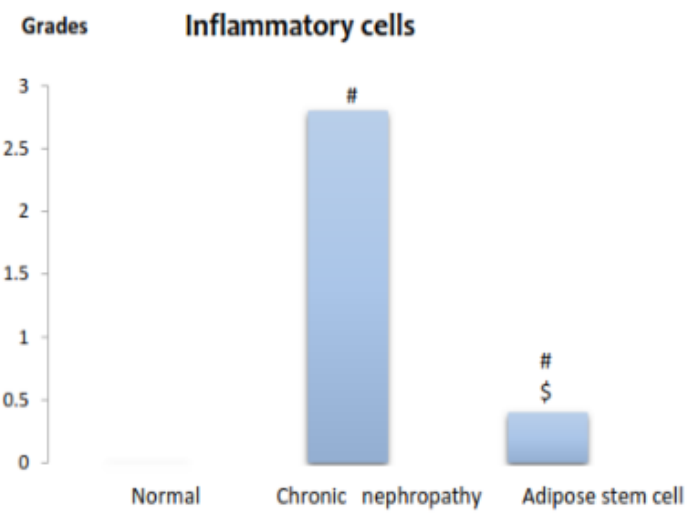

Figure 9: Histopathology scoring of renal tissue structure

(\#) significant $P$ value compared to normal group and (\$) significant $P$ value compared to Ch. nephropathy)
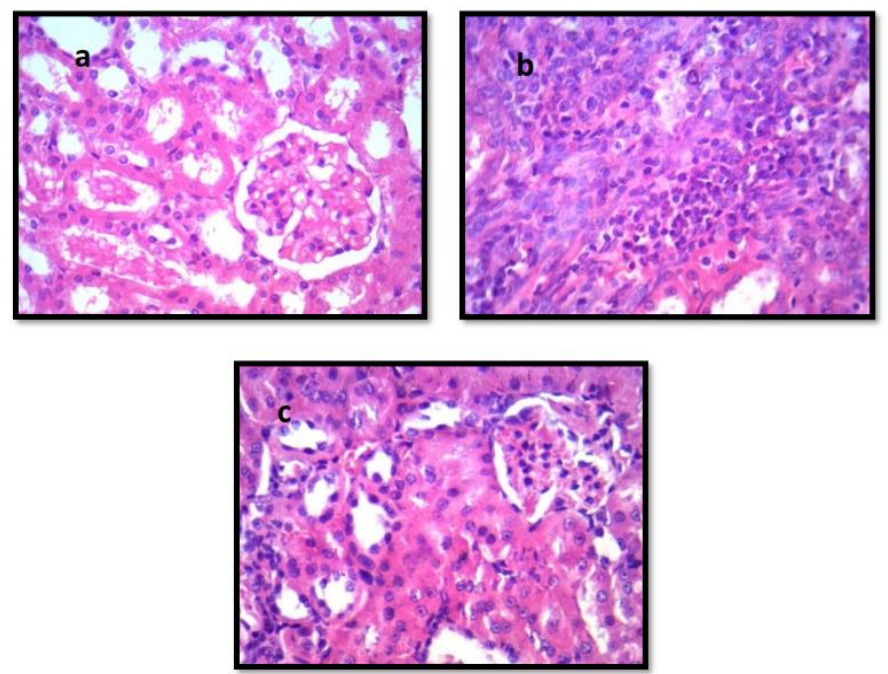

Figure 10: Hematoxylin and Eosin staining of renal tissue (x400) in the 3 groups a: normal, b: chronic nephropathy, c: ADSC group 

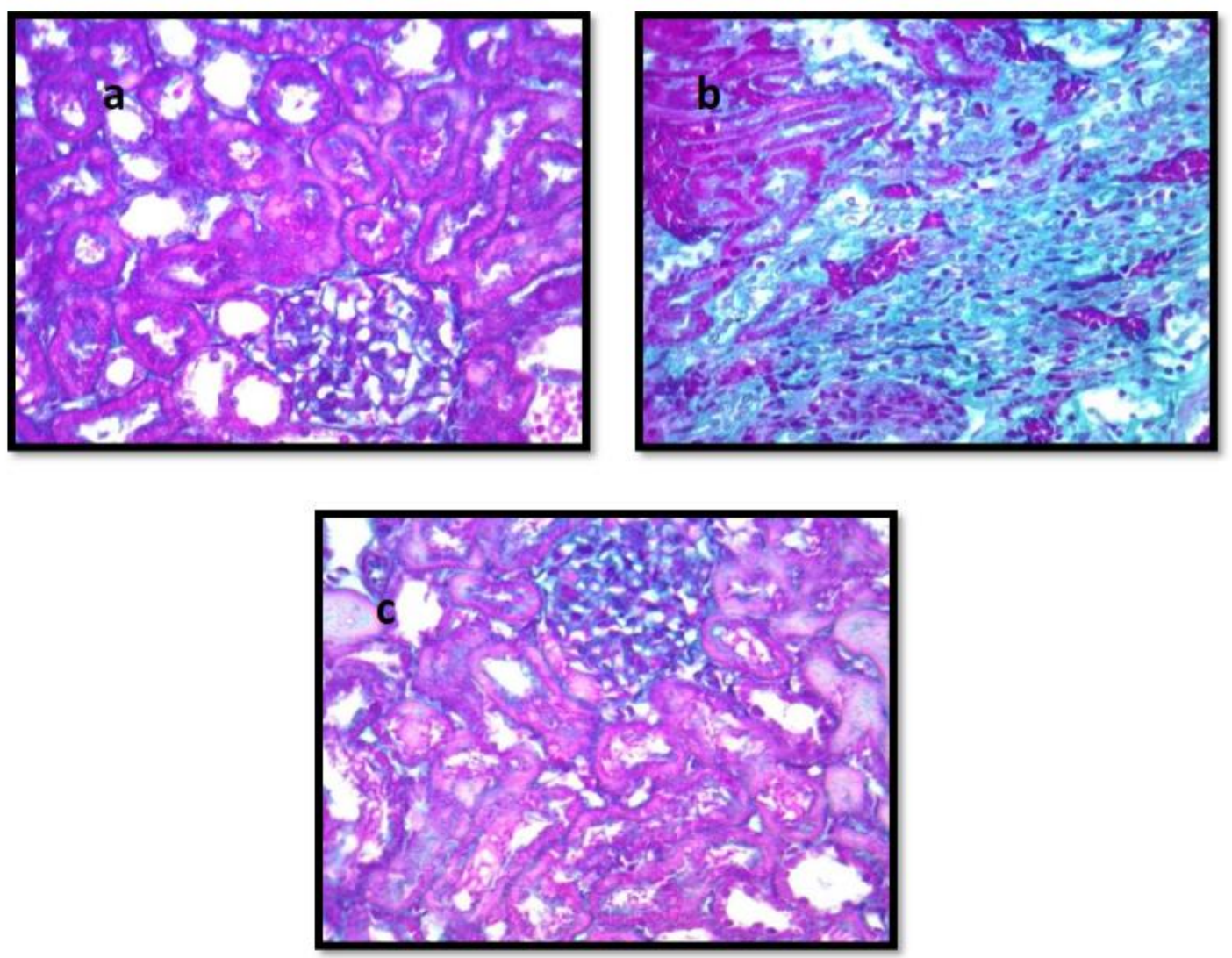

Figure 11: Masson's trichrome staining of renal tissue $x 400$ in 3 groups

Masson's trichrome staining of renal tissue in normal group showing showed thin fibrous stroma with no fibrosis, glomerular tuft, and renal tubule (figure 11a). Masson's trichrome staining of renal tissue in chronic nephropathy group showed moderate peritubular and perivascular fibrosis (figure 11b). Masson's trichrome staining of renal tissue in ADSC group showed improvement with no fibrosis, renal tubule, and glomerular tuft (figure 11c).

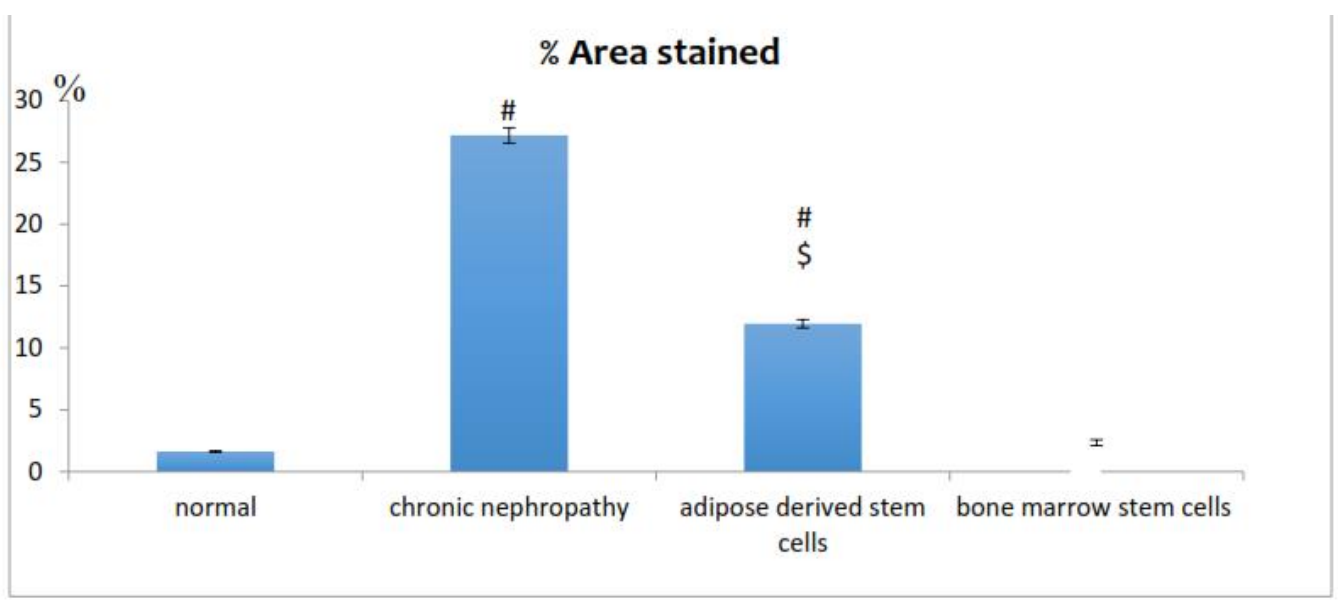

Figure 12: Percentage area stained for histopathology

(\#) significant $P$ value compared to normal group and (\$) significant $P$ value compared to Ch. nephropathy) 
a

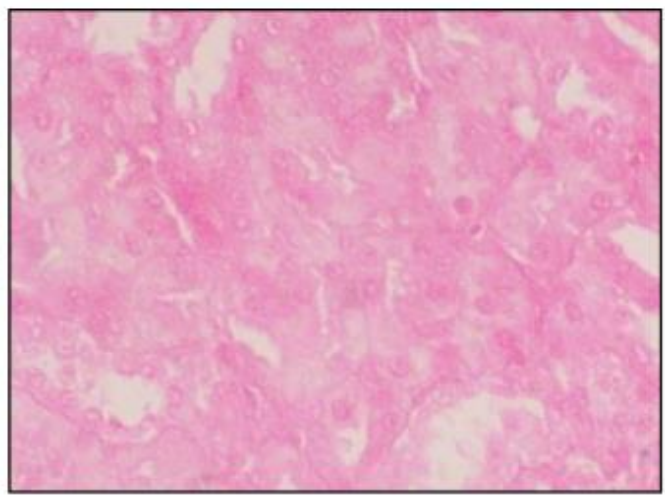

b

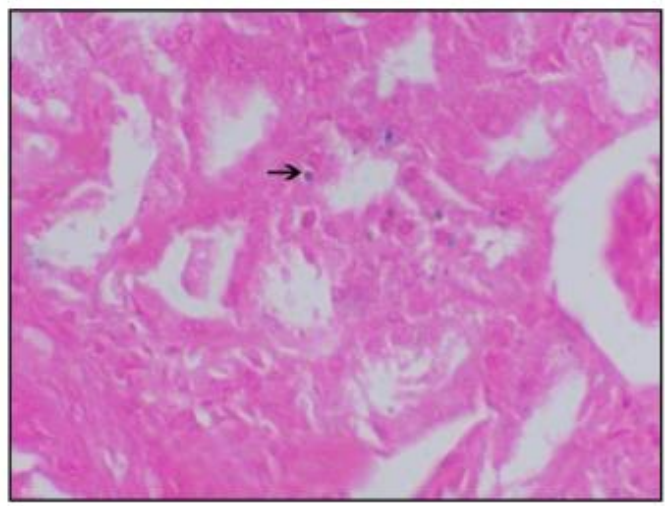

Figure 13: Prussian blue stain for stem cell homing X 100 of renal tissue

Prussian blue stain for stem cell homing in chronic nephropathy group showed negative staining (a). While adipose treated group showed positive staining for Prussian blue stain (bluish pigments within the tubular lining epithelium) (b).

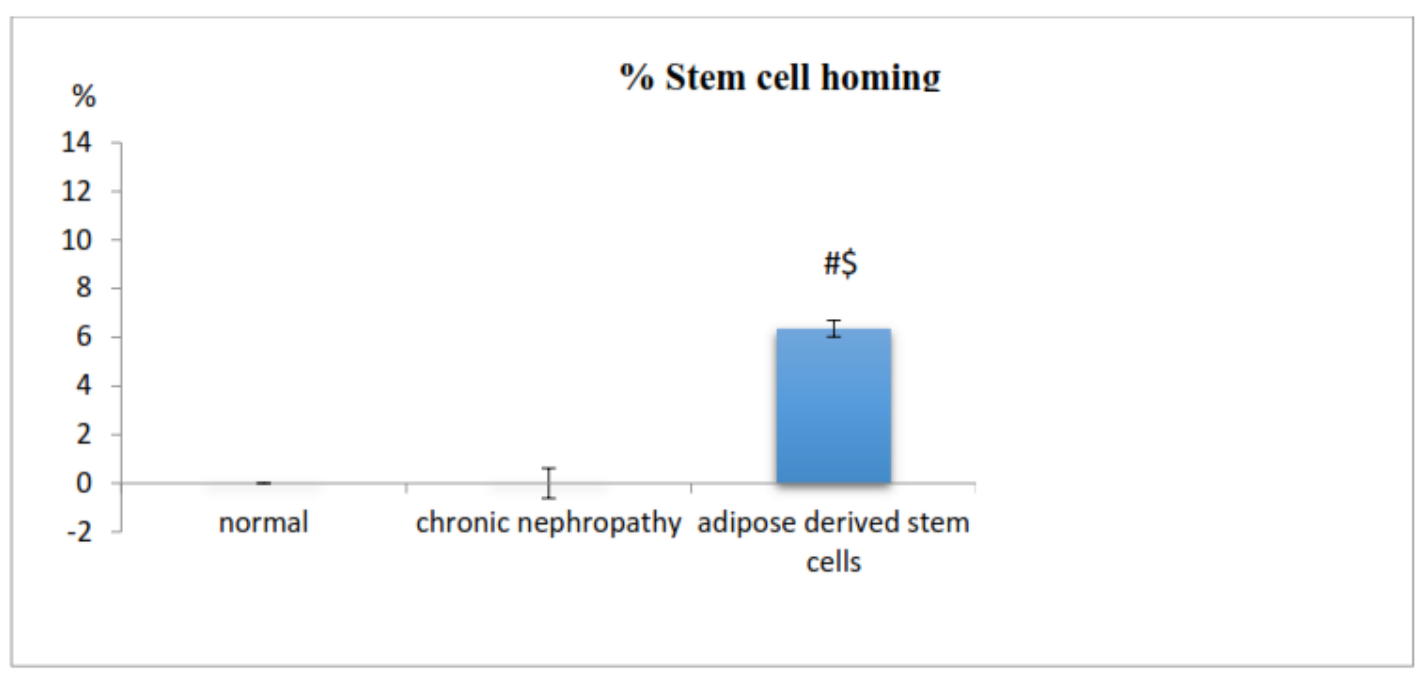

Figure 14: Percentage of stem cell homing in renal tissue

(*) significant $\mathrm{P}$ value in comparison to ADSC group.

\section{References}

1. Afifi A. (2008). Annual Reports of the Egyptian Renal Registry, 1996-2008 Cytotherapy.;8:315-7.

2. Isaac J, Spaeth E, Tsai TH et al. (2013) Improving Global Outcomes (KDIGO) CKD Work Group (2013) KDIGO clinical practice guideline for the evaluation and management of chronic kidney disease. Kidney. Int Suppl; 31:150-7.

3. Dominici $M$, Le BK, Mueller I, et al. (2006) Minimal criteria for defining multipotent mesenchymal stromal cells. The International Society for Cellular Therapy position statement. Cytotherapy; 8:315-7.

4. Togel F, Hu Z, Weiss K, et al. (2005) Administered mesenchymal stem cells protect against ischemic acute renal failure through differentiation-ind- 
ependent mechanisms. Am J Physiol Renal Physiol.;289: F31-42.

5. Klopp AH, Gupta A, Spaeth E, Andreeff $M$, Marini F. (2011) Concise review: Dissecting a discrepancy in the literature: do mesenchymal stem cells support or suppress tumor growth. Stem Cells; 29:11-9.

6. Chen YT, Sun CK, Lin YC, et al. (2011) Adipose-derived mesenchymal stem cell protects kidneys against ischemiareperfusion injury through suppressing oxidative stress and inflammatory reaction. J Transl Med; 9:51.

7. Nafar M, Sahraei Z, Salamzadeh J, Samavat S, Vaziri ND. (2011) Oxidative stress in kidney transplantation: causes, consequences, and potential treatment. Iran J Kidney Dis.; 5:357-72.

8. Zickri $M B$, Zaghloul S, Farouk $M$ and Abdel Fattah MM (2012): Effect of stem cell therapy on Adriamycin induced tubulointerstitial injury. Int J Stem Cells, pp. 130-139.

9. Anan $\mathrm{HH}$, Zidan RA, Shaheen $\mathrm{MA}$ and Abd-El Fattah EA (2016): Therapeutic efficacy of bone marrow derived mesenchymal stromal cells versus losartan on adriamycin-induced renal cortical injury in adult albino rats.18(8):970-84.

10. Nakia D, Jeffrey M, Mandi J, Lopez D. (2011). Mesenchymal Stromal Cells: Past, Present, and Future .First published: 11 January.

11. Musina RA, Bekchanova Es, Belyavskii Av, Griacako TS and Sukhikh GT (2007): Umbilical cord blood Mesenchymal stem cells. Bulletin of experimental Biology and Medicine vol. 143. No. 1.

12. Sarhan $M$, El Serougy $H$, Hussein $A M$ and El-dosoky (2014): Impact of bonemarrow-derived mesenchymal stem cells on adriamycin-induced chronic nephropathy. Canadian journal of physiology and pharmacology.92:9.

13. Dai R, Wang Z, Samanipour R, Koo Kl, Kim K. (2016): Stem Cells Int. 2016: 6737345. Doi: 10.1155/ 2016/ 6737345. Epub 2016

14. Lee Vincent \& Harris David. (2011). Adriamycin nephropathy: A model of focal segmental glomerulosclerosis. Nephrology (Carlton, Vic.). 16.30-8.10.1111/ j.1440-1797.2010.01383. x.

15. Choi SJ, Kim JK, Hwang SD (2010): Mesenchymal stem cell therapy for chronic renal failure. Expert Opin Biol Ther, 10:1217-1226.

16. Magnasco A, Corseli $M$, Bertelli R, et al. (2008): Mesenchymal stem cells protective effect in Adriamycin model of nephropathy. Cell transplantation 17, 10-11: 1157-1167

17. Jirkovsky E, Popelova O, KrivakovaStankova P, et al. (2012) Chronic Anthracycline cardiotoxicity: Molecular \& Functional analysis with focus on nuclear factor erythroid 2-related factor 2 and mitochondrial biogenesis pathways. J. Pharmacol Exp Ther 343:468478.

18. Binder BY, Gentos DC, Leach JK (2014): Lypophosphatidic acid protects human mesenchymal stem cells from differentiation-dependent vulnerability to apoptosis. Tissue Eng Part A; 20:11561164.

19. Dou L, Bertrand E, Cerini C, et al. (2004) The uremic solutes $p$-cresol and indoxyl sulfate inhibit endothelial proliferation and wound repair. Kidney Int. $65,442-451$

20. Lee SR, Lee SH, Moon JY, et al. (2010) Repeated administration of bone marrow-derived mesenchymal stem cells improved the protective effects on a remnant kidney model. Ren Fail, 32:840-848. 